\title{
Mean Square Convergence of Consensus Algorithms in Random WSNs
}

\author{
Silvana Silva Pereira, Student Member, IEEE, and Alba Pagès-Zamora, Member, IEEE
}

\begin{abstract}
Distributed consensus algorithms for estimation of parameters or detection of events in wireless sensor networks have attracted considerable attention in recent years. A necessary condition to achieve a consensus on the average of the initial values is that the topology of the underlying graph is balanced or symmetric at every time instant. However, communication impairments can make the topology vary randomly in time, and instantaneous link symmetry between pairs of nodes is not guaranteed unless an acknowledgment protocol or an equivalent approach is implemented. In this paper, we evaluate the convergence of the consensus algorithm in the mean square sense in wireless sensor networks with random asymmetric topologies. For the case of links with equal probability of connection, a closed form expression for the mean square error of the state along with the dynamical range and the optimum value of the link weights that guarantee convergence are derived. For the case of links with different probabilities of connection, an upper bound for the mean square error of the state is derived. This upper bound can be computed for any time instant and can be employed to compute a link weight that reduces the convergence time of the algorithm.
\end{abstract}

Index Terms-Asymmetric links, mean average consensus, mean square convergence, random topology, wireless sensor networks.

\section{INTRODUCTION}

$\mathbf{T}$ HE study of algorithms for wireless sensor networks (WSNs) has been motivated by military and civilian applications like battlefield surveillance, target tracking, environmental monitoring or home automation [1], [2]. Typically, WSNs include a more complex unit where the rest of the nodes convey their sensing measurements and which is often in charge of making the final decision. This unit is called the fusion center (FC). The nodes in these centralized networks require medium access control (MAC) and routing protocols to forward their data, and these protocols increase the complexity and the cost of the devices. Also, when emergency situations arise, the information flow of multiple simultaneous transmissions can create congestion around the FC. In addition, centralized networks are less robust to node failures and have limited scalability, since a

Manuscript received March 26, 2009; accepted January 19, 2010. Date of publication February 17, 2010; date of current version April 14, 2010. The associate editor coordinating the review of this manuscript and approving it for publication was Prof. Hongbin Li. This work has been funded in part by the European Commission (033914-WINSOC), the Spanish Ministry of Education and Science and FEDER funds (TEC2006-06481 and CONSOLIDER CSD2008-00010 COMONSENS) and the Catalan Government (2009SGR-01236).

The authors are with the Signal Processing and Communications Group (SPCOM), Universitat Politècnica de Catalunya-Barcelona Tech (UPC), C/Jordi Girona 1-3 08034, Barcelona, Spain (e-mail: silvana.silva@upc.edu; alba.pages@upc.edu).

Color versions of one or more of the figures in this paper are available online at http://ieeexplore.ieee.org.

Digital Object Identifier 10.1109/TSP.2010.2043140 complete reorganization must take place every time a node dies out or is added to the network. In networks with a decentralized structure however, the nodes organize themselves and are able to carry out the computations using only local information. Recent advances in sensing and DSP technology allow the manufacture of low cost devices of reduced size and typically with reduced communication capabilities. These simple nodes can implement low complexity algorithms and protocols to reduce the overall energy consumption [3].

Average consensus algorithms are low complexity iterative schemes where several nodes interact with each other to reach an agreement regarding the average value of an initial set of measurements (see [4]-[10]). The communications in a WSN are usually exposed to node and channel failures, changes in the environment and/or mobility of the nodes, which make the topology (connectivity) vary with time. Olfati-Saber and Murray introduce in [7] the concept of switching topology and show that a sufficient condition to solve the average consensus problem is that the instantaneous topology, which belongs to a finite set of known topologies, is balanced and strongly connected. Jadbabaie et al. in [11] use results on stochastic matrix theory to show that a sufficient condition for the system to converge to a common value asymptotically, which is not necessarily the average, is that the network is periodically connected. Ren and Beard in [12] generalize the results in [11] and show that a necessary and sufficient condition to achieve a consensus is that there is at least one node that can communicate with all the other nodes in a finite union of intervals.

When the topology is random, i.e., the existence of a link between a given pair of nodes is random, the convergence of the consensus algorithm can be characterized in probabilistic terms. Hatano and Mesbahi in [14] use notions on stochastic stability to study the convergence in probability over random graphs, where the links failures are modeled as independent identically distributed (i.i.d.) random variables (r.v.'s). Kar and Moura in [15] relate mean square convergence of the consensus algorithm to the second smallest eigenvalue of the average Laplacian matrix, and use semi-definite convex programming in [16] to propose a probabilistic topology in order to maximize the convergence rate. In their further work in [17], they propose two different models to reduce either the bias or the variance produced by the noise in the consensus value. In all these contributions, the instantaneous topology is assumed balanced or symmetric, i.e., the Laplacian matrix is always positive semidefinite, to ensure that the network converges to the average of the initial values. Note that in a practical implementation of the consensus algorithm, symmetric links can be guaranteed implementing for instance an acknowledgment protocol to ensure reciprocal information exchange at each iteration. 
For networks with nonsymmetric random topologies, Wu in [18] uses results from inhomogeneous Markov chains to derive sufficient conditions for convergence in probability. TahbazSalehi and Jadbabaie use ergodicity properties in [19] to show almost sure convergence to a common value, and relate it to the second largest eigenvalue of the average weight matrix. Porfiri and Stilwell in [20] show that consensus is asymptotically achieved in random directed graphs almost surely if the probability that the network is strongly connected is nonzero, and this occurs whenever the expected network is strongly connected. A similar approach is presented in [21], where the authors show almost sure local synchronization of oscillators in a random weighted directed graph. Zhou and Wang in [22] redefine and derive the asymptotic and per-step convergence factors from [6] for random directed networks. Rabbat et al. show in [23] that the average consensus is asymptotically achieved in both symmetric and nonsymmetric topologies provided that the value of the link weights tends to zero, at the cost of increasing the convergence time.

In this paper we study the convergence in the mean square sense of the algorithm in [7] for WSN with random asymmetric topologies, evaluated with respect to the statistical mean of the initial measurements. The constraint on instantaneous link symmetry is relaxed and only symmetric probability of connection is assumed. In order to speed up the convergence, we consider link weights not tending to zero and constant throughout the running. For links with equal probability of connection, a closed-form expression for the mean square error (MSE) of the state is derived along with the dynamical range and the link weight minimizing the convergence time. For the case of links with different probabilities of connection, an upper bound for the MSE and a procedure to compute a link weight that reduces the convergence time are derived. The paper is organized as follows. In Section II we introduce some basic graph theory concepts for random topologies. In Section III we present the consensus algorithm for networks with random topologies and in Section IV we analyze the convergence of the algorithm in the mean square sense. Section V includes the analysis for the case of links with equal probability of connection, the convergence conditions and the asymptotic MSE. Section VI includes the study for the case of different probabilities of connection among the nodes. The simulation results and the conclusions for the paper are included in Sections VII and VIII, respectively.

\section{GRAPH THEORY CONCEPTS}

The information flow among the nodes of a network with random topology can be described by a directed graph (digraph) $\mathcal{G}(k)=\{\mathcal{V}, \mathcal{E}(k)\}$, where $\mathcal{E}(k)$ is the set of edges (links) $e_{i j}$ at time $k$ for all $i, j=\{1, \cdots, N\}$, such that the information flows from node $j$ to node $i$, and $\mathcal{V}$ is the constant set of vertices (nodes) [24]. The randomness of the connections is modeled assigning a given independent probability to each link, such that $e_{i j} \in \mathcal{E}(k)$ with probability $0 \leq p_{i j} \leq 1$, and each link has a weight $a_{i j}(k)$ equal to 1 if $e_{i j} \in \mathcal{E}(k)$ and equal to 0 otherwise. We assume no multiple edges or loops, i.e., $a_{i i}(k)=0 \forall k$, and links with symmetric probability of connection $p_{i j}=p_{j i}$ $\forall\{i, j\}$. The set of neighbors of node $i$ at time $k$ is denoted
$\mathcal{N}_{i}(k)=\left\{j \in \mathcal{V}: e_{i j} \in \mathcal{E}(k)\right\}$ and represents the set of nodes sending information to node $i$. If $\sum_{j=1}^{N} a_{i j}(k)=\sum_{j=1}^{N} a_{j i}(k)$, we say that the $i^{\text {th }}$ node is balanced. A digraph $\mathcal{G}(k)$ with all its nodes balanced is a balanced digraph. A path in a digraph is a sequence of alternating nodes and edges that starts and ends at different nodes. A digraph is strongly connected if any node can be reached from any other node of the graph by a directed path. $\mathbf{P} \in \mathbb{R}^{N \times N}$ denotes the connection probability matrix with entries $[\mathbf{P}]_{i j}=p_{i j}$ and $[\mathbf{P}]_{i i}=0$. The instantaneous adjacency matrix $\mathbf{A}(k) \in \mathbb{R}^{N \times N}$ of $\mathcal{G}(k)$ with connection probability matrix $\mathbf{P}$ is random with statistically independent entries given by

$$
[\mathbf{A}(k)]_{i j}= \begin{cases}1 & \text { with probability } p_{i j} \\ 0 & \text { with probability } 1-p_{i j}\end{cases}
$$

and symmetric expected value matrix $\mathbb{E}[\mathbf{A}(k)]=\overline{\mathbf{A}}=\mathbf{P}$. The degree matrix $\mathbf{D}(k) \in \mathbb{R}^{N \times N}$ is a diagonal matrix whose entries are $[\mathbf{D}(k)]_{i i}=\sum_{j=1}^{N}[\mathbf{A}(k)]_{i j}$, and the instantaneous Laplacian is defined as $\mathbf{L}(k) \stackrel{=}{=} \mathbf{D}(k)-\mathbf{A}(k)$. Due to the random nature of $\mathbf{A}(k)$, the instantaneous Laplacian $\mathbf{L}(k)$ is random. By construction, the smallest eigenvalue of $\mathbf{L}(k)$ in magnitude ${ }^{1}$ is $\lambda_{1}(\mathbf{L}(k))=0$ and has associated right eigenvector $\mathbf{1} \in \mathbb{R}^{N \times 1}$, an all-ones vector of length $N$. If $\mathcal{G}(k)$ is strongly connected, $\lambda_{1}(\mathbf{L}(k))$ has algebraic multiplicity one and $\mathbf{L}(k)$ is an irreducible matrix [26]. $\overline{\mathcal{G}}$ denotes the expected graph, i.e., the average graph over time. $\overline{\mathcal{G}}$ has symmetric associated Laplacian $\overline{\mathbf{L}}=\overline{\mathbf{D}}-\mathbf{P}$. For additional concepts in algebraic graph theory and random directed graphs, see [25] and [27].

\section{CONSENSUS IN RANDOM TOPOLOGIES}

Consider a WSN composed of $N$ nodes indexed with $i=$ $\{1, \cdots, N\}$ and a scalar value $x_{i}(k)$ defined as the state of node $i$ at time $k \geq 0$. Each node is programmed to run a discrete dynamical system whose state is initialized at time $k=0$ with the value of a single measurement, and updated iteratively using a linear combination of its own previous value and the information received from its neighbors as follows:

$$
x_{i}(k)=x_{i}(k-1)+\epsilon \sum_{j \in \mathcal{N}_{i}(k)}\left(x_{j}(k-1)-x_{i}(k-1)\right)
$$

where the link weight $\epsilon$ is chosen to satisfy convergence conditions. Let $\mathbf{x}(k) \in \mathbb{R}^{N \times 1}$ denote the vector of all states at time $k$. For a network with random topology, the evolution of $\mathbf{x}(k)$ can be written in matrix form as follows:

$$
\mathbf{x}(k)=\mathbf{W}(k-1) \mathbf{x}(k-1), \quad \forall k>0
$$

where the weight matrix $\mathbf{W}(k) \in \mathbb{R}^{N \times N}$ is random and modeled as

$$
\mathbf{W}(k)=\mathbf{I}-\epsilon \mathbf{L}(k), \quad \forall k \geq 0
$$

I is the identity matrix and $\epsilon$ is equal for all the iterations. The authors in [28] propose a link weight $\epsilon$ decreasing in time in order to reduce the variance produced by noise in the consensus value, and a similar approach is proposed in [17]. Moreover, the

\footnotetext{
${ }^{1}$ To be consistent with graph theory notation, the eigenvalues of the Laplacian matrix only are arranged in increasing order.
} 
authors in [20] study the consensus with link weights that are not necessarily positive. In our model however, $\epsilon$ is assumed equal for all the links, constant and positive. The dynamical range of $\epsilon$ is basic for the convergence analysis and will be determined later. We assume throughout the analysis that the expected network $\overline{\mathcal{G}}$ is undirected and connected, such that $\overline{\mathbf{L}}$ is irreducible. For convenience, we define the following set of matrices:

Definition 1: The set

$$
\mathcal{W}=\{\mathbf{W}(k) \mid \mathbf{W}(k)=\mathbf{I}-\epsilon \mathbf{L}(k), \forall k \geq 0\}
$$

is composed of the random matrices generated by (3) with $\mathbf{A}(k)$ as defined in (1), for all possible topologies such that $\mathbb{E}[\mathbf{L}(k)]=$ $\overline{\mathbf{L}}$ is irreducible.

Note that the matrices in $\mathcal{W}$ are independent with one eigenvalue equal to one and associated right eigenvector 1 . The matrices $\{\mathbf{W}(k), \forall k\}$ in (2) belong to $\mathcal{W}$ and are therefore independent of each other with one eigenvalue equal to one and associated right eigenvector 1 . The associated left eigenvector varies randomly from realization to realization because $\mathbf{W}(k)$ is, in general, nonsymmetric.

The evolution of the state vector $\mathbf{x}(k)$ in (2) can be rewritten as follows:

$$
\mathbf{x}(k)=\mathbf{M}_{\mathbf{w}}(k) \mathbf{x}(0)
$$

where $\mathbf{x}(0)=\left[x_{1}(0) x_{2}(0) \cdots x_{N}(0)\right]^{T}$ is the vector of initial measurements and the matrix

$$
\mathbf{M}_{\mathbf{w}}(k)=\prod_{l=1}^{k} \mathbf{W}(k-l), \quad \forall k>0
$$

is assumed independent of $\mathbf{x}(0)$ for all $k$. The properties of this matrix will be used in the subsequent analysis, and are stated below in Lemma 1.

Lemma 1:

a. The product matrix $\mathbf{M}_{\mathbf{w}}(k)$ defined in (6) satisfies $\mathbf{M}_{\mathbf{w}}(k) \mathbf{1}=\mathbf{1}$ for all $k$.

b. The expected value matrix

$$
\mathbb{E}\left[\mathbf{M}_{\mathbf{w}}(k)\right]=\overline{\mathbf{W}}^{k}
$$

is symmetric and double-stochastic.

Proof:

a. The first property of $\mathbf{M}_{\mathbf{w}}(k)$ is easily demonstrated since the matrices in the set $\mathcal{W}$ defined in (4) have at least one eigenvalue equal to one with associated right eigenvector 1.

b. The equality in (7) follows from the independence assumption of the matrices in the set $\mathcal{W}$ and the fact that the expected value of $\mathbf{W}(k)$ is given by $\overline{\mathbf{W}}=\mathbf{I}-\epsilon \overline{\mathbf{L}}$. Further, since $\mathbf{P}$ is assumed symmetric, $\overline{\mathbf{L}}$ and $\overline{\mathbf{W}}$ are symmetric. $\overline{\mathbf{W}}$ is therefore a double-stochastic matrix with eigenvalues $\lambda_{i}(\overline{\mathbf{W}})=\mathbf{I}-\epsilon \lambda_{i}(\overline{\mathbf{L}})$ for all $i \in\{1, \cdots, N\}$.

Assume a set of initial measurements modeled as i.i.d. Gaussian r.v.'s with mean $x_{m}$ and variance $\sigma_{0}^{2}$, such that $\mathbb{E}[\mathbf{x}(0)]=x_{m} \mathbf{1}$ and $\mathbb{E}\left[\mathbf{x}(0) \mathbf{x}(0)^{T}\right]=\sigma_{0}^{2} \mathbf{I}+x_{m}^{2} \mathbf{1 1}^{T}$. Due to the random nature of both $\mathbf{x}(0)$ and the matrices $\{\mathbf{W}(k), \forall k\}$ in (2), we consider the convergence of $\mathbf{x}(k)$ in (5) to the mean average consensus, defined as

$$
\mathbf{x}_{m a}=\frac{1}{N} \mathbf{1}^{T} \mathbb{E}[\mathbf{x}(0)] \mathbf{1}=x_{m} \mathbf{1}
$$

From Lemma 1.a. we know that $\mathbf{M}_{\mathbf{w}}(k)$ has one eigenvalue equal to one with associated right eigenvector 1 . For those realizations for which $\mathbf{M}_{\mathbf{w}}(k)$ is also primitive with $\lambda_{1}\left(\mathbf{M}_{\mathbf{w}}(k)\right)=$ 1 , we have that

$$
\lim _{k \rightarrow \infty} \mathbf{x}(k)=\frac{1}{N} \mathbf{1}_{\mathbf{M}} \mathbf{x}(0)
$$

where $\gamma_{\mathbf{M}}$ is the left eigenvector associated with the eigenvalue one of $\mathbf{M}_{\mathbf{w}}(k)$ for $k \rightarrow \infty$. Recalling the independence assumption of $\mathbf{M}_{\mathbf{w}}(k)$ and $\mathbf{x}(0)$ in (5), and using Lemma 1.b. it is not difficult to check that

$$
\lim _{k \rightarrow \infty} \mathbb{E}[\mathbf{x}(k)]=\mathbf{x}_{m a}
$$

showing that the estimation is unbiased. Moreover, from the assumption of connectivity in average and the results in [23], we know that as the value of $\epsilon$ approaches zero, the system asymptotically reaches the average consensus, which coincides in this case with the ML (maximum likelihood) estimator. In general, as the value of $\epsilon$ decreases, the convergence time increases. Since we focus on reducing the convergence time of the algorithm, we consider a value of $\epsilon$ not tending to zero, although at the cost of deviating from the average consensus. The main contribution of this paper is both the evaluation of this deviation (for certain statistical properties of the connection probability matrix), and the impact of the value of $\epsilon$ on this deviation. Some of the results contained in this work were developed in [29] and are included here for the sake of completeness. In Section IV, we analyze the MSE of the state vector $\mathbf{x}(k)$ in (5) with respect to the mean average consensus defined in (8). In Section V we address the case of an Erdôs-Rényi random graph model [30], where the probability of having a link between any two nodes is the same for all pairs of nodes, and later in Section VI we consider the case where these probabilities are allowed to be different.

\section{Mean SQuare Convergence}

Let's define the MSE of the state with respect to the mean average consensus as follows:

$$
\operatorname{MSE}(x(k))=\frac{1}{N} \mathbb{E}\left[\left\|\mathbf{x}(k)-\mathbf{x}_{m a}\right\|_{2}^{2}\right] .
$$

Substituting (5) in (9) and expanding the expression yields

$$
\begin{aligned}
& \operatorname{MSE}(x(k))=\frac{1}{N} \mathbb{E}\left[\mathbf{x}(0)^{T} \mathbf{M}_{\mathbf{w}}^{T}(k) \mathbf{M}_{\mathbf{w}}(k) \mathbf{x}(0)\right. \\
& \left.-\mathbf{x}(0)^{T} \mathbf{M}_{\mathbf{w}}^{T}(k) \mathbf{x}_{m a}-\mathbf{x}_{m a}^{T} \mathbf{M}_{\mathbf{w}}(k) \mathbf{x}(0)+\mathbf{x}_{m a}^{T} \mathbf{x}_{m a}\right] .
\end{aligned}
$$

For convenience, consider the matrix

$$
\mathbf{R}_{\mathbf{w}}(k)=\mathbb{E}\left[\mathbf{M}_{\mathbf{w}}^{T}(k) \mathbf{M}_{\mathbf{w}}(k)\right]
$$

whose most important property is stated in the following lemma: 
Lemma 2: The matrix $\mathbf{R}_{\mathrm{w}}(k)$ in (11) is double-stochastic.

Proof: The matrix $\mathbf{R}_{\mathrm{w}}(k)$ is symmetric due to its definition in (11). To show that it is double-stochastic, we just need to show that it is row-stochastic, i.e.,

$$
\begin{aligned}
\mathbf{R}_{\mathbf{w}}(k) \mathbf{1} & =\mathbb{E}\left[\mathbf{M}_{\mathbf{w}}^{T}(k) \mathbf{M}_{\mathbf{w}}(k) \mathbf{1}\right] \\
& =\mathbb{E}\left[\mathbf{M}_{\mathbf{w}}^{T}(k) \mathbf{1}\right]=\left(\overline{\mathbf{W}}^{k}\right)^{T} \mathbf{1}=\mathbf{1}
\end{aligned}
$$

where the second and the last equalities hold because of Lemma 1.a. and 1.b. respectively.

Using (11) and (7), and recalling the independence of $\mathbf{M}_{\mathbf{w}}(k)$ and $\mathbf{x}(0)$, the expression in (10) can be rewritten as

$$
\begin{aligned}
& \operatorname{MSE}(x(k))=\frac{1}{N}( \operatorname{tr}\left(\left(\sigma_{0}^{2} \mathbf{I}+x_{m}^{2} \mathbf{1 1}^{T}\right) \cdot \mathbf{R}_{\mathbf{w}}(k)\right) \\
&-x_{m} \mathbf{1}^{T}\left(\overline{\mathbf{W}}^{k}\right)^{T} \mathbf{x}_{m a} \\
&\left.-x_{m} \mathbf{x}_{m a}^{T} \overline{\mathbf{W}}^{k} \mathbf{1}+\mathbf{x}_{m a}^{T} \mathbf{x}_{m a}\right) \\
&=\frac{\sigma_{0}^{2}}{N} \cdot \operatorname{tr}\left(\mathbf{R}_{\mathbf{w}}(k)\right)
\end{aligned}
$$

where $\operatorname{tr}($.$) stands for the trace operator. The expression in$ (12) tells us that the MSE at every node will be deviated from the variance of the ML estimator, i.e., $\sigma_{0}^{2} / N$, by a factor $\operatorname{tr}\left(\mathbf{R}_{\mathbf{w}}(k)\right)$. In order to characterize this deviation we start expanding the expression of $\mathbf{R}_{\mathrm{w}}(k)$ in (11). Applying the linearity of the trace and the expected value operators and using the fact that $\{\mathbf{W}(k), \forall k\} \in \mathcal{W},(12)$ can be expressed as follows:

$$
\begin{aligned}
\operatorname{MSE}(x(k))=\frac{\sigma_{0}^{2}}{N} \operatorname{tr}\left(\mathbb { E } \left[\mathbf{W}^{T}(1) \ldots \mathbf{W}^{T}(k-1)\right.\right. \\
\\
\times \mathbf{W}(k-1) \ldots \mathbf{W}(1)] \\
\left.\times \mathbb{E}\left[\mathbf{W}(0) \mathbf{W}^{T}(0)\right]\right) \\
=\frac{\sigma_{0}^{2}}{N} \cdot \operatorname{tr}\left(\mathbf{R}_{\mathbf{w}}(k-1) \cdot \mathbf{C}_{\mathbf{w}}\right)
\end{aligned}
$$

where $\mathbf{C}_{\mathbf{w}}=\mathbb{E}\left[\mathbf{W}(k) \mathbf{W}^{T}(k)\right] \forall k$. After some matrix manipulations, $\mathbf{C}_{\mathbf{w}}$ can be expressed analytically as

$$
\mathbf{C}_{\mathbf{w}}=\mathbf{I}-2 \epsilon \overline{\mathbf{L}}+\epsilon^{2} \mathbb{E}\left[\mathbf{L}(k) \mathbf{L}(k)^{T}\right]
$$

where $\overline{\mathbf{L}}$ and $\mathbb{E}\left[\mathbf{L}(k) \mathbf{L}(k)^{T}\right]$ have entries given by

$$
[\overline{\mathbf{L}}]_{i j}= \begin{cases}\sum_{l=1}^{N} p_{i l} & i=j \\ -p_{i j} & i \neq j\end{cases}
$$

$$
\begin{aligned}
& \mathbb{E}\left[\mathbf{L}(k) \mathbf{L}(k)^{T}\right]_{i j} \\
& = \begin{cases}2 \sum_{l=1}^{N} p_{i l}+\sum_{l=1}^{N} \sum_{\substack{m=1 \\
m \neq l}}^{N} p_{i l} p_{i m} & i=j \\
-p_{i j}\left(\sum_{l=1}^{N} p_{i l}+\sum_{l=1}^{N} p_{j l}\right)+\sum_{\substack{l=1 \\
l \neq j}}^{N} p_{i l} p_{j l} & i \neq j\end{cases}
\end{aligned}
$$

The next step is to analyze the MSE expression in (13) in two different situations: when all the links have the same probability of connection, and when the links have different, although symmetric, probability of connection.

\section{MSE ANALYSIS With EQUAL LINK PROBABILITIES}

We assume an Erdős-Rényi random graph, where for any pair of nodes $\{i, j\} \in \mathcal{V}$, the communication link between them exists with probability $p_{i j}=p_{j i}=p$. The following theorem resumes our first important result [29]:

Theorem 1: Consider the iterative algorithm in (2) with $N$ nodes, probability of connection $0 \leq p \leq 1$ equal for all the links and i.i.d. initial values $\mathbf{x}(0)$ with mean $x_{m}$ and variance $\sigma_{0}^{2}$. The $\operatorname{MSE}(x(k))$ averaged over $N$ nodes in (9) is equal to

$$
\operatorname{MSE}(x(k))=\sigma_{0}^{2}\left(\frac{b}{1-a+b}-\frac{(a-1)}{1-a+b}(a-b)^{k}\right)
$$

where

$$
\begin{aligned}
& a=1-2(N-1) \epsilon+\left(2(N-1) p+(N-1)(N-2) p^{2}\right) \epsilon^{2} \\
& b=2 p \epsilon-N p^{2} \epsilon^{2} .
\end{aligned}
$$

Proof: Replacing for $p$ in (15) and in (16), and substituting the resulting expressions in (14), we get

$$
\mathbf{C}_{\mathbf{w}}=b \cdot 11^{T}+\mathbf{I}(a-b)
$$

with $a$ and $b$ as defined in (18). Then, replacing (19) in (13), the MSE expression becomes

$$
\operatorname{MSE}(x(k))=\frac{\sigma_{0}^{2}}{N}\left(N b+(a-b) \operatorname{tr}\left(\mathbf{R}_{\mathbf{w}}(k-1)\right)\right)
$$

where we have used the properties of $\mathbf{R}_{\mathbf{w}}(k)$ in Lemma 2. Substituting the trace above recursively and noting that $\operatorname{tr}\left(\mathbf{R}_{\mathbf{w}}(1)\right)=\operatorname{tr}\left(\mathbf{C}_{\mathbf{w}}\right)$ we obtain

$$
\begin{aligned}
\frac{\sigma_{0}^{2}}{N} \operatorname{tr}\left(\mathbf{R}_{\mathbf{w}}(k)\right) & =\frac{\sigma_{0}^{2}}{N} \cdot N\left(\sum_{l=0}^{k-2} b(a-b)^{l}+a(a-b)^{k-1}\right) \\
& =\sigma_{0}^{2}\left(\frac{b}{1-a+b}-\frac{(a-1)}{1-a+b}(a-b)^{k}\right)
\end{aligned}
$$

and the proof is completed.

The expression in (17) allows us to compute the MSE of the state at any time instant off-line, as it only requires knowledge of the general parameters $N, p$ and $\epsilon$. In order to provide a better understanding of this expression, in the following subsections we study the convergence conditions of the MSE expression derived in Theorem 1 and the residual MSE after convergence. For a deeper insight into different forms for convergence of stochastic systems, the reader can refer to [31] and [32].

\section{A. MSE Convergence and Optimal Choice of $\epsilon$}

Since the convergence of the MSE defined in (9) is related to the value of $\epsilon$, we aim now at determining the dynamical range of $\epsilon$, finding the value that maximizes the convergence rate and evaluating the convergence time. We observe that the convergence of (17) is related to the term $(a-b)$. For simplicity, consider the following function:

Definition 2: For a given number of nodes $N>0$ and probability of connection $0 \leq p \leq 1$, define the function

$$
f(\epsilon)=a-b
$$


where $a$ and $b$ are as defined in (18).

The convergence time of the MSE in (17) is therefore proportional to the term

$$
r=-\frac{1}{\ln f(\epsilon)}
$$

Clearly, a necessary and sufficient condition for the product $(f(\epsilon))^{k}$ to approach zero as $k \rightarrow \infty$ is that $|f(\epsilon)|<1$. Observe that, for $N>0$ and $0 \leq p \leq 1, f(\epsilon)$ is a quadratic nonnegative function with $f(0)=1$. The optimum $\epsilon$ can therefore be easily determined, as stated in the following lemma:

Lemma 3: For a given number of nodes $N$ and a given probability of connection $0 \leq p \leq 1$, the value of $\epsilon$ that minimizes the convergence time of the MSE in (17) is given by

$$
\epsilon^{*}=\frac{N}{2(N-1)+(N-1)^{2} p+p} .
$$

Proof: The value of $\epsilon^{*}$ in (22) corresponds to the minimum of a convex nonnegative quadratic function, namely $f(\epsilon)$ in (20), so the demonstration is straightforward.

Substituting for $\epsilon^{*}$ in (21), the convergence rate of the algorithm is proportional to

$$
r=-\ln ^{-1}\left(\frac{2(N-1)(1-p)}{2(N-1)+(N-1)^{2} p+p}\right) .
$$

It is interesting to note that, as the network size increases, the value of $\epsilon^{*}$ approaches zero and the convergence rate of the algorithm is faster. The impact of the network size on the convergence time is more evident for low values of $p$ because, as $p$ approaches 1 , the consensus value is reached in a single iteration. The results obtained from (20) and (22) allow us to bound the values of $\epsilon$ for convergence, as stated in the following theorem:

Theorem 2: Consider the iterative algorithm in (2) with $N$ nodes, probability of connection $0 \leq p \leq 1$ equal for all the links and i.i.d. initial values $\mathbf{x}(0)$ with mean $x_{m}$ and variance $\sigma_{0}^{2}$. Then, $\mathbf{x}(k)$ converges in the mean square sense if

$$
0<\epsilon<\frac{2 N}{2(N-1)+(N-1)^{2} p+p} .
$$

Proof: The demonstration follows from Lemma 3 and the fact that $f(\epsilon)$ is a quadratic nonnegative function with $f(0)=1$ and vertex in $\epsilon^{*}$.

Theorem 2 resumes an important result. It states that if we choose $\epsilon$ belonging to the interval defined in (24), we can guarantee that as $k \rightarrow \infty$, the algorithm in (2) converges in the mean square sense to a consensus. In addition, choosing the link weights equal to the value $\epsilon^{*}$ in (22), the convergence time is minimized and proportional to (23).

Remark that under assumptions of equal probability of connection for all the links, the expected Laplacian $\overline{\mathbf{L}}$ has one eigenvalue zero and $(N-1)$ eigenvalues equal to $N p$. As the network size increases or as $p$ approaches 1 , the upper bound of $\epsilon$ in (24) approaches the value $2 / N$, coinciding with the upper bound of $\epsilon$ in a fully connected network, as expected. In that case $\epsilon^{*}=2 /\left(\lambda_{2}(\overline{\mathbf{L}})+\lambda_{N}(\overline{\mathbf{L}})\right)=1 / N$, and we obtain the same result using (22). According to the results in [19] (Corollary 4), a necessary and sufficient condition for almost sure convergence of the consensus algorithm in (2) is that the second largest eigenvalue of the average weight matrix satisfies $\left|\lambda_{2}(\overline{\mathbf{W}})\right|<1$, assuming that the matrices in the set $\{\mathbf{W}(k), \forall k\}$ have positive diagonal entries. In our model however, we do not restrict the weight matrices to have positive diagonal entries, since that condition would require $\epsilon<(1 /(N-1))$. In [16, Lemma 11], the authors state that for a network connected in average over time, the value of $\epsilon$ that minimizes the convergence time of (2) in the mean square sense belongs to the interval $0<\epsilon<\left(2 / \lambda_{2}(\overline{\mathbf{L}})\right)$, which in our case reads $0<\epsilon<(2 / N p)$. However, the value of $\epsilon^{*}$ is not specified in [16], whereas in Lemma 3 we provide this optimum value when the links have equal probability of connection. In fact, it can be checked that $\epsilon^{*}$ in (22) belongs to the interval derived in [16].

\section{B. Asymptotic MSE}

The purpose of this subsection is to determine the impact of the network size and the probability of connection on the asymptotic MSE. Choosing $\epsilon$ belonging to the interval in (24), the limit of the MSE as $k \rightarrow \infty$ is given by

$$
\begin{aligned}
\lim _{k \rightarrow \infty} \operatorname{MSE}(x(k)) \\
\quad=\sigma_{0}^{2}\left(\frac{b}{1-a+b}\right) \\
\quad=\frac{\sigma_{0}^{2}}{N}\left(\frac{2 N-N^{2} p \epsilon}{2 N-\left(2(N-1)+(N-1)^{2} p+p\right) \epsilon}\right) \\
\quad=\frac{\sigma_{0}^{2}}{N} \cdot g(\epsilon)
\end{aligned}
$$

where we have substituted for the values of $a$ and $b$. Clearly, the function $g(\epsilon)$ in (25) approaches 1 as $\epsilon \rightarrow 0$, so the MSE at each node tends to $\sigma_{0}^{2} / N$ as the value of $\epsilon \rightarrow 0 .{ }^{2}$ Therefore, $g(\epsilon)$ provides the deviation of the MSE with respect to the optimum $\sigma_{0}^{2} / N$. Actually, it can be seen that this deviation increases monotonically for $\epsilon \in\left(0,2 \epsilon^{*}\right)$ and tends to infinity as $\epsilon \rightarrow 2 \epsilon^{*}$. In order to gain intuitive insight on the impact of $N$ and $p$ on the limit in (25), we assume that $\epsilon$ is sufficiently small to approximate $g(\epsilon)$ using a first-order Taylor series expansion. Noting that $g(0)=1$ and $g^{\prime}(0)=((N-1) / N)(1-p)$, in the vicinity of $\epsilon=0$, the limit in (25) behaves as

$$
\lim _{k \rightarrow \infty} \operatorname{MSE}(x(k)) \approx \frac{\sigma_{0}^{2}}{N}\left(1+\frac{N-1}{N}(1-p) \epsilon\right) .
$$

This result shows that for small values of $\epsilon$, the impact of $N$ on the deviation of the asymptotic MSE with respect to $\sigma_{0}^{2} / N$ becomes negligible after a relatively high number of nodes. On the other hand, the higher the probability of connection of the links is, the closer the asymptotic MSE is to the benchmark $\sigma_{0}^{2} / N$.

\section{MSE Evaluation With Different Link Probabilities}

In this section we evaluate the MSE expression in (13) when the communication links have different probabilities of connec-

\footnotetext{
${ }^{2}$ This result is compliant with [23].
} 
tion. At this point we make use of a theorem stated in [33], included below as a lemma.

Lemma 4: Inequality for the trace of matrix product-For any matrix $\mathbf{A} \in \mathbb{R}^{N \times N}$ and any symmetric matrix $\mathbf{B} \in \mathbb{R}^{N \times N}$, let $\widehat{\mathbf{A}}=\left(\mathbf{A}+\mathbf{A}^{T}\right) / 2$. Then

$$
\operatorname{tr}(\mathbf{A B}) \leq \lambda_{1}(\widehat{\mathbf{A}}) \operatorname{tr}(\mathbf{B})-\lambda_{N}(\mathbf{B})\left(N \lambda_{1}(\widehat{\mathbf{A}})-\operatorname{tr}(\mathbf{A})\right)
$$

where $\lambda_{1}($.$) and \lambda_{N}($.$) denote largest and smallest eigenvalue,$ respectively.

Proof: See [33, Theorem 3].

Using Lemma 4 we can resume our next relevant result in the following theorem.

Theorem 3: Consider the consensus algorithm in (2) for $N$ nodes with symmetric nonnegative connection probability matrix $\mathbf{P}$ and i.i.d. initial values $\mathbf{x}(0)$ with mean $x_{m}$ and variance $\sigma_{0}^{2}$. Assuming that the largest eigenvalue of $\mathbf{R}_{\mathbf{w}}(k)$ in (11) is equal to $1 \forall k$, the $\operatorname{MSE}(x(k))$ averaged over $N$ nodes in (9) is upper bounded by

$$
\operatorname{MSE}(x(k)) \leq \frac{\sigma_{0}^{2}}{N}\left(N+\left(\operatorname{tr}\left(\mathbf{C}_{\mathbf{w}}\right)-N\right) \frac{1-\lambda_{N}^{k}\left(\mathbf{C}_{\mathbf{w}}\right)}{1-\lambda_{N}\left(\mathbf{C}_{\mathbf{w}}\right)}\right)
$$

where $\mathbf{C}_{\mathbf{w}}$ is the matrix defined in (14) and $\lambda_{N}\left(\mathbf{C}_{\mathbf{w}}\right)$ denotes its smallest eigenvalue.

Proof: Applying the trace inequality in (26), the trace in (13) is upper bounded by

$\operatorname{tr}\left(\mathbf{R}_{\mathbf{w}}(k)\right) \leq \operatorname{tr}\left(\mathbf{C}_{\mathbf{w}}\right)-\lambda_{N}\left(\mathbf{C}_{\mathbf{w}}\right) N+\lambda_{N}\left(\mathbf{C}_{\mathbf{w}}\right) \operatorname{tr}\left(\mathbf{R}_{\mathbf{w}}(k-1)\right)$

where we have substituted for $\mathbf{A}=\mathbf{R}_{\mathbf{w}}(k-1)$ and $\mathbf{B}=\mathbf{C}_{\mathbf{w}}$ in (26), and assumed that $\lambda_{1}\left(\mathbf{R}_{\mathrm{w}}(k-1)\right)=1$ (see Lemma 2). Replacing $\operatorname{tr}\left(\mathbf{R}_{\mathbf{w}}(k-1)\right)$ for $\operatorname{tr}\left(\mathbf{R}_{\mathrm{w}}(k-2) \cdot \mathbf{C}_{\mathbf{w}}\right)$ above, and computing the upper bound recursively until reaching $\mathbf{R}_{\mathbf{w}}(1)$ we obtain

$$
\begin{aligned}
\operatorname{tr}\left(\mathbf{R}_{\mathbf{w}}(k)\right) \leq \operatorname{tr}\left(\mathbf{C}_{\mathbf{w}}\right)+\operatorname{tr}\left(\mathbf{C}_{\mathbf{w}}\right) \sum_{i=1}^{k-2} \lambda_{N}^{i}\left(\mathbf{C}_{\mathbf{w}}\right) \\
\quad+\lambda_{N}^{k-1}\left(\mathbf{C}_{\mathbf{w}}\right) \operatorname{tr}\left(\mathbf{R}_{\mathbf{w}}(1)\right)-N \sum_{i=1}^{k-1} \lambda_{N}^{i}\left(\mathbf{C}_{\mathbf{w}}\right) .
\end{aligned}
$$

This inequality can be further simplified to

$$
\begin{aligned}
\operatorname{tr}\left(\mathbf{R}_{\mathbf{w}}(k)\right) & \leq \operatorname{tr}\left(\mathbf{C}_{\mathbf{w}}\right)+\left(\operatorname{tr}\left(\mathbf{C}_{\mathbf{w}}\right)-N\right) \sum_{i=1}^{k-1} \lambda_{N}^{i}\left(\mathbf{C}_{\mathbf{w}}\right) \\
& =N+\left(\operatorname{tr}\left(\mathbf{C}_{\mathbf{w}}\right)-N\right) \sum_{i=0}^{k-1} \lambda_{N}^{i}\left(\mathbf{C}_{\mathbf{w}}\right),
\end{aligned}
$$

and replacing for the trace in (13) the proof is completed.

Theorem 3 states that, for a known connection probability matrix $\mathbf{P}$, we can find $\mathbf{C}_{\mathbf{w}}$ using (14), compute its eigenvalues and then compute the upper bound for the MSE in (27) for any time instant. It can be checked that when the link probabilities are all equal, the value of the upper bound in (27) coincides with the exact MSE expression in (17). This is due to the particular structure of $\mathbf{C}_{\mathbf{w}}$ when substituting $p_{i j}=p \forall\{i, j\}$. It is important to remark that Theorem 3 provides a bound for the estimation error of the consensus algorithm whenever the matrix
$\mathbf{R}_{\mathbf{w}}(k)$ has largest eigenvalue equal to one. This is a strong condition and in the following subsection we give sufficient conditions on the value of $\epsilon$ to guarantee both that $\mathbf{R}_{\mathrm{w}}(k)$ has largest eigenvalue $\lambda_{1}\left(\mathbf{R}_{\mathbf{w}}(k)\right)=1 \forall k$, and that the MSE upper bound in (27) converges.

\section{A. Asymptotic MSE Upper Bound and Optimal Choice of $\epsilon$}

We observe that the term $\lambda_{N}^{k}\left(\mathbf{C}_{\mathbf{w}}\right)$ on the right-hand side of the inequality in (27) tends to zero as $k$ increases. Therefore, the MSE upper bound in (27) converges whenever $\left|\lambda_{N}\left(\mathbf{C}_{\mathbf{w}}\right)\right|<1$. In order to analyze the asymptotic behavior of the upper bound, we make use of the following Lemma:

Lemma 5: Consider the matrix $\mathbf{C}_{\mathbf{w}}$ defined in (14) with symmetric connection probability matrix $\mathbf{P}$. If $\epsilon \in(0,1 /(N-1)]$, the smallest eigenvalue of $\mathbf{C}_{\mathbf{w}}$ satisfies

$$
0 \leq \lambda_{N}\left(\mathbf{C}_{\mathbf{w}}\right)<1, \quad \forall 0 \leq p_{i j} \leq 1 .
$$

Proof: The left inequality in (28) holds because $\mathbf{C}_{\mathbf{w}}=$ $\mathbb{E}\left[\mathbf{W}(k) \mathbf{W}^{T}(k)\right]$ is a real, symmetric and positive semi-definite matrix, and therefore its eigenvalues are all real and nonnegative. To prove the right inequality in (28) we have that

$$
\operatorname{tr}\left(\mathbf{C}_{\mathbf{w}}\right)=\sum_{i=1}^{N} \lambda_{i}\left(\mathbf{C}_{\mathbf{w}}\right) \geq N \lambda_{N}\left(\mathbf{C}_{\mathbf{w}}\right),
$$

therefore, the smallest eigenvalue of $\mathbf{C}_{\mathbf{w}}$ is upper bounded by

$$
\begin{aligned}
\lambda_{N}\left(\mathbf{C}_{\mathbf{w}}\right) \leq & \frac{1}{N} \sum_{i=1}^{N}\left[\mathbf{C}_{\mathbf{w}}\right]_{i i} \\
= & 1-\frac{2 \epsilon}{N} \sum_{i=1}^{N} \sum_{l=1}^{N} p_{i l} \\
& +\frac{\epsilon^{2}}{N}\left(\sum_{i=1}^{N} \sum_{l=1}^{N} p_{i l}\left(2+\sum_{\substack{m=1 \\
m \neq l}}^{N} p_{i m}\right)\right)
\end{aligned}
$$

where we have replaced for the components $\left[\mathbf{C}_{\mathbf{w}}\right]_{i i}$ substituting (15) and (16) in (14). For simplicity, we denote the expression on the right-hand side of (29) as the function $h(\epsilon)$. This function satisfies $h(0)=1$ and, after some algebraic manipulations we have

$$
\begin{aligned}
h\left(\frac{1}{N-1}\right)= & 1+\frac{1}{(N-1)^{2}} \\
& \times \sum_{i=1}^{N} \sum_{l=1}^{N} p_{i l}\left(-2(N-2)+\sum_{\substack{m=1 \\
m \neq l}}^{N} p_{i m}\right) \\
\leq & 1-\frac{N-2}{N(N-1)^{2}} \sum_{i=1}^{N} \sum_{l=1}^{N} p_{i l}<1
\end{aligned}
$$

where the first inequality holds because $\sum_{\substack{m=1 \\ m \neq l}}^{N} p_{i m} \leq(N-2)$ and the second inequality holds because we assume $p_{i j}>0$ for some $\{i, j\}$. Since $h(\epsilon)$ above is a convex function on $\epsilon$, we have that

$$
h(\epsilon)<1, \quad \forall \epsilon \in\left(0, \frac{1}{N-1}\right] .
$$


Substituting (30) in (29) we prove the right inequality of (28).

Lemma 5 shows that choosing $\epsilon$ in the interval in (30), the smallest eigenvalue of $\mathbf{C}_{\mathbf{w}}$ is less than one in magnitude, so the term $\lambda_{N}^{k}\left(\mathbf{C}_{\mathbf{w}}\right)$ tends to zero as $k \rightarrow \infty$. Note that this dynamical range also guarantees that the weight matrices $\{\mathbf{W}(k), \forall k\}$ in (2) are nonnegative with positive diagonal entries. In that case, it can be shown that the matrix $\mathbf{R}_{\mathrm{w}}(k)$ in (11) is nonnegative for all $k$, and due to Corollary 8.1.30 in [26], it has largest eigenvalue one with algebraic multiplicity one. Thus, the upper bound in (27) applies and converges for $\epsilon \in(0,1 /(N-1)]$ and in addition, $\mathbf{x}(k)$ converges almost surely to $\mathbf{x}_{m a}$ by the results in [19].

Applying the results from Lemma 5, the asymptotic MSE in (27) is upper bounded by

$$
\lim _{k \rightarrow \infty} \operatorname{MSE}(\mathbf{x}(k)) \leq \sigma_{0}^{2}\left(\frac{\operatorname{tr}\left(\mathbf{C}_{\mathbf{w}}\right)-N \lambda_{N}\left(\mathbf{C}_{\mathbf{w}}\right)}{1-\lambda_{N}\left(\mathbf{C}_{\mathbf{w}}\right)}\right) .
$$

The expression above gives an upper bound for the error after the algorithm has converged, and this upper bound can be computed off-line, since the matrix $\mathbf{C}_{\mathbf{w}}$ depends on the probability matrix $\mathbf{P}$ and on the constant $\epsilon$.

Optimum $\epsilon$-In Lemma 5 we have shown that if $\epsilon$ belongs to the range $(0,1 /(N-1)]$, the assumptions made in Theorem 3 are valid and the upper bound in (27) converges. Clearly, a smaller value of $\lambda_{N}\left(\mathbf{C}_{\mathbf{w}}\right)$ would lead to a faster convergence of the upper bound. In order to maximize the convergence rate of the upper bound and since $\lambda_{N}\left(\mathbf{C}_{\mathbf{w}}\right)$ is not convex on $\epsilon$, we propose to select the value of $\epsilon$ that minimizes $\lambda_{N}\left(\mathbf{C}_{\mathbf{w}}\right)$ using an exhaustive search. In general, the optimum $\epsilon^{*}$ is outside the dynamical range $(0,1 /(N-1)]$ that guarantees the result of Theorem 3. However, as illustrated in the simulations, the choice of $\epsilon^{*}$ provides convergence of the empirical MSE in addition to fast convergence of the algorithm.

\section{SimULATIONS}

The analytical results obtained in Sections V and VI are supported here with computer simulations. We simulate a WSN randomly deployed in a squared area of $100 \times 100$ units where the communication links are random. The entries of the vector $\mathbf{x}(0)$ are modeled as Gaussian r.v.'s with mean $x_{m}=20$ and variance $\sigma_{0}^{2}=5$. A total of 100000 independent realizations were run to obtain the empirical MSE, where the position of the nodes and the connection probability matrix $\mathbf{P}$ are kept fixed for all the realizations, while a new Laplacian matrix is generated in each iteration and realization.

In the first set of simulations, we let $N=20$ and equal probability $p=0.4$ for all the links. Fig. 1 shows the empirical MSE of the state in $\mathrm{dB}$ with respect to the mean average defined in (9), along with the theoretical MSE expression in (17) (patterns) for three different cases: $\epsilon^{*}$ found using (22) and equal to 0.1094 (dotted-line), $\epsilon=1 /(N-1)=0.0526$ (dashed-line) and $\epsilon=0.2<2 \epsilon^{*}$ (dashed-dotted line). The benchmark value $\sigma_{0}^{2} / N \approx-6$ is included in solid line. As expected, the empirical values obtained with (9) match the theoretical values obtained with (17). We observe that the gap corresponding to the term $g(\epsilon)$ defined in (25) decreases with $\epsilon$. However, choosing the optimum $\epsilon^{*}$ we achieve fastest convergence of the MSE (dotted-line) as stated by Lemma 3, whereas

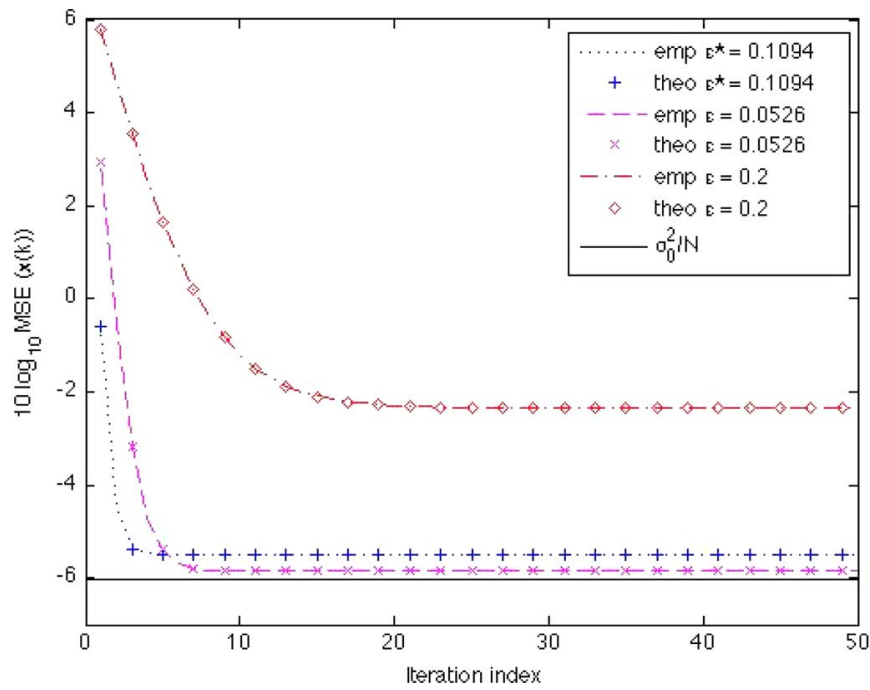

Fig. 1. Empirical and theoretical $\operatorname{MSE}(x(k))$ as a function of $k$ in $\mathrm{dB}$ averaged over $N=20$ nodes with $p=0.4$ and different values of $\epsilon$.

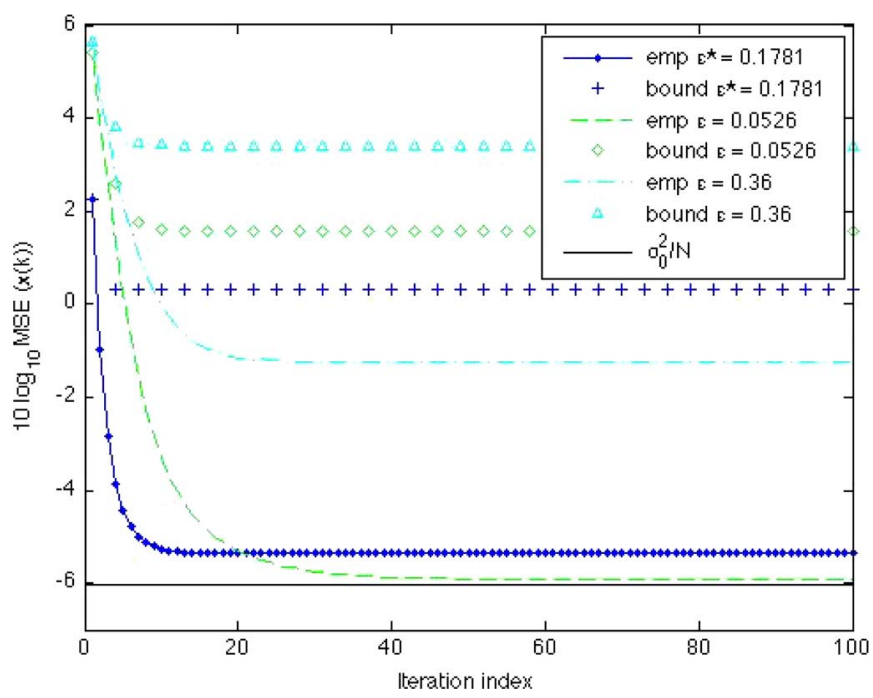

Fig. 2. Empirical $\operatorname{MSE}(x(k))$ and theoretical upper bound as a function of $k$ in $\mathrm{dB}$ averaged over all the nodes for a small-world network with $N=20,4$ nearest neighbors, connection probability 0.4 and different values of $\epsilon$.

choosing the smallest $\epsilon$ the curve is closer to the benchmark (dashed-line).

In the second set of simulations we consider different probabilities of connection for the links. Specifically, we simulate three different deployments: a small-world network [34] with $N=20,4$ nearest neighbors and shortcut probability 0.4 , and a uniform random network [35] with $N=20$ and $N=100$ nodes respectively. The nonzero entries of the matrix $\mathbf{P}$ in the small-world network are set equal to $p=0.4$, whereas for the uniform random deployment they are modeled as i.i.d. r.v.'s uniformly distributed between 0 and 1. Fig. 2 shows the empirical MSE of the state in $\mathrm{dB}$ for the small-world network, and Figs. 3 and 4 show the empirical MSE for the random network of 20 and 100 nodes respectively. The theoretical upper bound computed with (27) is depicted with patterns. Three different 


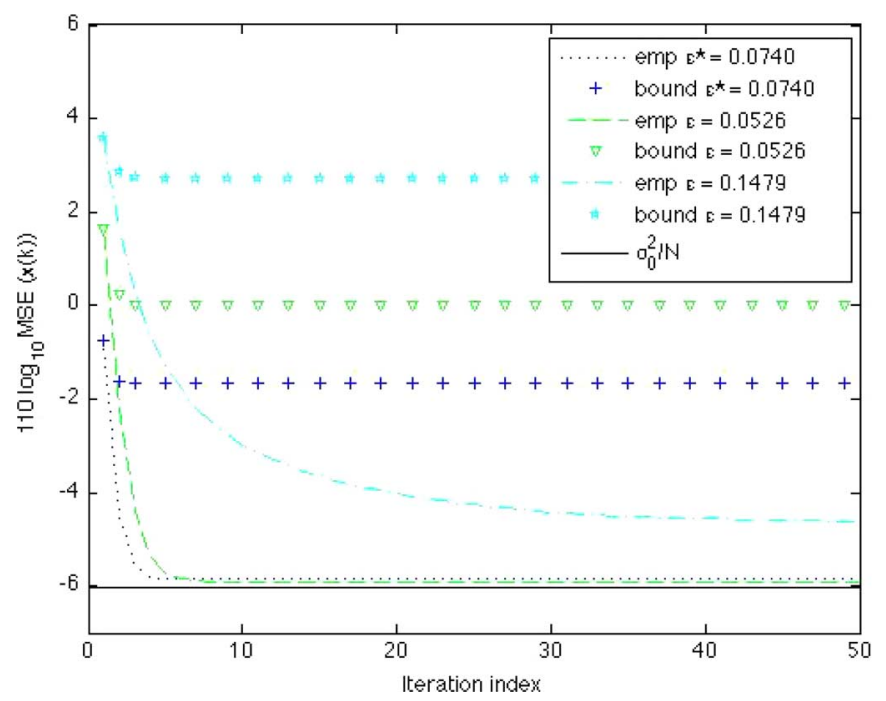

Fig. 3. Empirical $\operatorname{MSE}(x(k))$ and theoretical upper bound as a function of $k$ in $\mathrm{dB}$ averaged over $N=20$ nodes for different probabilities of connection and different values of $\epsilon$.

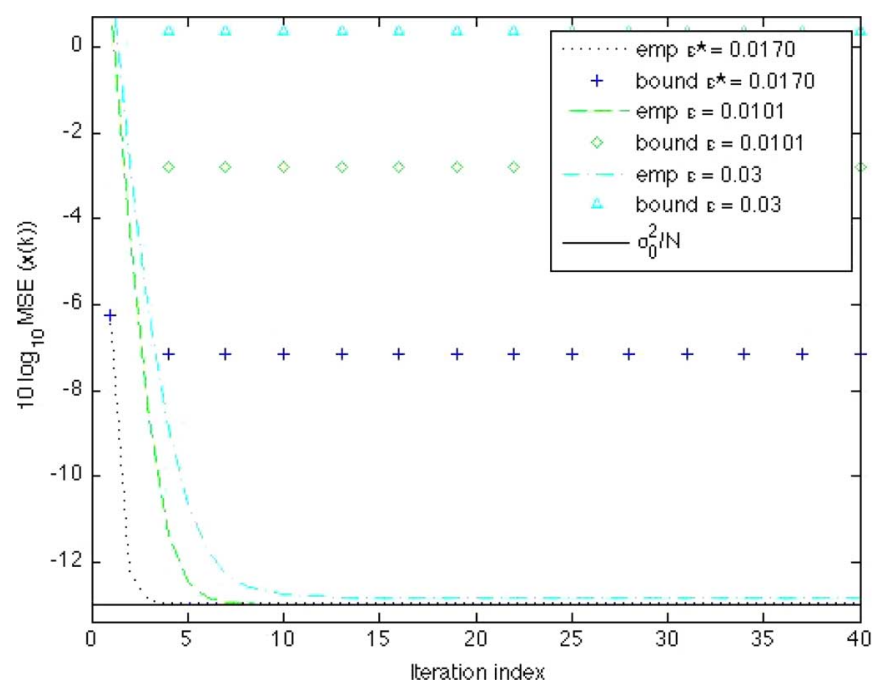

Fig. 4. Empirical $\operatorname{MSE}(x(k))$ and theoretical upper bound as a function of $k$ in $\mathrm{dB}$ averaged over $N=100$ nodes for different probabilities of connection and different values of $\epsilon$.

values of $\epsilon$ were tested for each deployment, all of them satisfying $\lambda_{N}\left(\mathbf{C}_{\mathbf{w}}\right)<1$. For the small-world network, we obtained $\epsilon^{*}=0.1781$ and for the random networks we obtained $\epsilon^{*}=0.0740$ and $\epsilon^{*}=0.0170$ respectively (dotted-dashed lines), using an exhaustive search to minimize $\lambda_{N}\left(\mathbf{C}_{\mathbf{w}}\right)$. The remaining choices of $\epsilon$ correspond to the values $1 /(N-1)$ (dashed-lines) and $\epsilon \approx 2 \epsilon^{*}$ (dashed-dotted line). The benchmark values $\sigma_{0}^{2} / N$ are included in solid line. Although the upper bound curves for the two smallest values of $\epsilon$ in all cases converge quite fast, the curve for $\epsilon^{*}$ converges faster. We observe that the curves for the empirical MSE behave rather similar to the upper bound curves, since the empirical MSE converges also faster when the optimum $\epsilon^{*}$ is chosen.

As previously stated, all the values of $\epsilon$ that give a $\lambda_{N}\left(\mathbf{C}_{\mathbf{w}}\right)<$ 1 , guarantee the convergence of the upper bound but not necessarily the convergence of the empirical MSE. In particular, in our simulations we have observed that if $\epsilon$ is larger than $2 \epsilon^{*}$, the MSE diverges despite the fact that $\lambda_{N}\left(\mathbf{C}_{\mathbf{w}}\right)$ still is lower than 1. For instance, with $N=20$ and $\epsilon=0.2$ (a figure is not included) the upper bound converges but the empirical MSE diverges. This is due to the fact that the eigenvalue equal to one of the matrix $\mathbf{R}_{\mathbf{w}}(k)$ is no longer the largest, and therefore the upper bound in (27) does not apply in this case.

\section{CONCLUSION}

We have shown that in a WSN with random topology and instantaneous asymmetric links consensus can be reached in the mean square sense, and the MSE of the state vector with respect to the mean average consensus can be characterized analytically with knowledge of the probability of connection of the links and the statistics of the initial measurements. For the case of links with equal probability of connection, a closed form expression for the MSE of the state, as well as the dynamical range of the link weight $\epsilon$ that guarantees mean square convergence of the algorithm have been derived. In addition, the optimum $\epsilon^{*}$ providing fastest convergence rate has been obtained. The MSE expression proves to be useful to characterize the convergence time of the consensus algorithm. For the case of links with different probabilities of connection, an upper bound for the MSE of the state has been derived. Although the upper bound differs from the empirical MSE, it can be employed for the computation of a link weight that provides a fast convergence of the algorithm to a consensus.

\section{REFERENCES}

[1] C.-Y. Chong and S. Kumar, "Sensor networks: Evolution, opportunities, and challenges," Proc. IEEE, vol. 91, no. 8, pp. 1247-1256, Aug. 2003.

[2] I. Akyildiz, W. Su, Y. Sankarasubramaniam, and E. Cayirci, "A survey on sensor networks," IEEE Commun. Mag., vol. 40, no. 8, pp. 102-114, Aug. 2002.

[3] D. P. Bertsekas and J. N. Tsitsiklis, Parallel and Distributed Computation: Numerical Methods. Englewood Cliffs, NJ: Prentice-Hall, 1997.

[4] R. Olfati-Saber, J. Fax, and R. Murray, "Consensus and cooperation in networked multi-agent systems," Proc. IEEE, vol. 95, no. 1, pp. 215-233, Jan. 2007.

[5] W. Ren, R. Beard, and E. Atkins, "A survey of consensus problems in multi-agent coordination," in Proc. Amer. Control Conf., Jun. 2005, vol. 3, pp. 1859-1864.

[6] L. Xiao and S. Boyd, "Fast linear iterations for distributed averaging," in Proc. 42nd IEEE Conf. Decision Control, Dec. 2003, vol. 5, pp. 4997-5002.

[7] R. Olfati-Saber and R. Murray, "Consensus problems in networks of agents with switching topology and time-delays," IEEE Trans. Autom. Control, vol. 49, no. 9, pp. 1520-1533, Sep. 2004.

[8] D. Scherber and H. Papadopoulos, "Locally constructed algorithms for distributed computations in ad hoc networks," in 3rd Int. Symp. Information Processing Sensor Networks (IPSN), Apr. 26-27, 2004, pp. 11-19.

[9] S. Barbarossa and G. Scutari, "Decentralized maximum-likelihood estimation for sensor networks composed of nonlinearly coupled dynamical systems," IEEE Trans. Signal Process., vol. 55, no. 7, pp. 3456-3470, 2007.

[10] I. D. Schizas, I. D. Schizas, G. B. Giannakis, S. I. Roumeliotis, and A. Ribeiro, "Consensus in ad hoc wsns with noisy links-Part ii: Distributed estimation and smoothing of random signals," IEEE Trans. Signal Process., vol. 56, no. 4, pp. 1650-1666, 2008.

[11] A. Jadbabaie, J. Lin, and A. Morse, "Coordination of groups of mobile autonomous agents using nearest neighbor rules," IEEE Trans. Autom. Control, vol. 48, no. 6, pp. 988-1001, Jun. 2003.

[12] W. Ren and R. Beard, "Consensus seeking in multiagent systems under dynamically changing interaction topologies," IEEE Trans. Autom. Control, vol. 50, no. 5, pp. 655-661, May 2005. 
[13] L. Moreau, "Stability of multiagent systems with time-dependent communication links," IEEE Trans. Autom. Control, vol. 50, no. 2, pp. 169-182, Feb. 2005.

[14] Y. Hatano and M. Mesbahi, "Agreement over random networks," in Proc. 43rd IEEE Conf. Decision Control (CDC), Dec. 2004, vol. 2, pp. 2010-2015.

[15] S. Kar and J. Moura, "Distributed average consensus in sensor networks with random link failures," in Proc. IEEE Int. Conf. Acoustics, Speech, Signal Processing (ICASSP), Apr. 15-20, 2007, vol. 2, pp. II-1013-II-1016.

[16] S. Kar and J. Moura, "Sensor networks with random links: Topology design for distributed consensus," IEEE Trans. Signal Process., vol. 56, no. 7, pp. 3315-3326, Jul. 2008.

[17] S. Kar and J. Moura, "Distributed consensus algorithms in sensor networks with imperfect communication: Link failures and channel noise," IEEE Trans. Signal Process., vol. 57, no. 1, pp. 355-369, Jan. 2009.

[18] C. W. Wu, "Synchronization and convergence of linear dynamics in random directed networks," IEEE Trans. Autom. Control, vol. 51, no. 7, pp. 1207-1210, 2006.

[19] A. Tahbaz-Salehi and A. Jadbabaie, "A necessary and sufficient condition for consensus over random networks," IEEE Trans. Autom. Control, vol. 53, no. 3, pp. 791-795, 2008.

[20] M. Porfiri and D. Stilwell, "Consensus seeking over random weighted directed graphs," IEEE Trans. Autom. Control, vol. 52, no. 9, pp. 1767-1773, 2007.

[21] M. Porfiri, D. J. Stilwell, and E. M. Bollt, "Synchronization in random weighted directed networks," IEEE Trans. Circuits Syst., vol. 55, no. 10, pp. 3170-3177, 2008 .

[22] J. Zhou and Q. Wang, "Convergence speed in distributed consensus over dynamically switching random networks," Automatica, vol. 45, no. 6, pp. 1455-1461, Jun. 2009.

[23] M. Rabbat, R. Nowak, and J. Bucklew, "Generalized consensus computation in networked systems with erasure links," in Proc. IEEE 6th Workshop Signal Processing Advances Wireless Communications (SPAWC), Jun. 2005, pp. 1088-1092.

[24] C. Godsil and G. Royle, Algebraic Graph Theory. Berlin, Germany: Springer-Verlag, 2001, vol. 207, Graduate Texts in Mathematics.

[25] M. Fiedler, "Algebraic connectivity of graphs," Czech. Math. J., vol. 23, no. 98, 1973.

[26] R. Horn and C. Johnson, Matrix Analysis. Cambridge, U.K.: Cambridge Univ. Press, 2006.

[27] C. W. Wu, "Algebraic connectivity of directed graphs," Linear Multilinear Algebra vol. 53, no. 3, pp. 203-223, 2005 [Online]. Available: http://dx.doi.org/10.1080/03081080500054810

[28] Y. Hatano, A. Das, and M. Mesbahi, "Agreement in presence of noise: Pseudogradients on random geometric networks," in Proc. 44th IEEE Conf. Decision Control Eur. Control Conf. (CDC-ECC), 2005, pp. 6382-6387.

[29] S. S. Pereira and A. Pagès-Zamora, "Fast mean square convergence of consensus algorithms in WSNs with random topologies," in Proc. IEEE Int. Conf. Acoustics, Speech, Signal Processing (ICASSP), 2009, pp. 2213-2216.

[30] P. Erdős and A. Rényi, "On the evolution of random graphs," Acta Mathematica Academiae Scientiarum, vol. 5, pp. 17-61, 1960.
[31] H. Kushner, Introduction to Stochastic Control. New York: Rinehart Winston, 1971.

[32] X. Mao, Exponential Stability of Stochastic Differential Equations. New York: Marcel Dekker, 1994.

[33] Y. Fang, K. Loparo, and X. Feng, "Inequalities for the trace of matrix product," IEEE Trans. Autom. Control, vol. 39, no. 12, pp. 2489-2490, Dec. 1994.

[34] D. J. Watts and S. H. Strogatz, "Collective dynamics of 'small-world' networks," Nature, vol. 393, no. 6684, pp. 440-442, Jun. 1998.

[35] B. Bollobás, Random Graphs, 2nd ed. Cambridge, U.K.: Cambidge Univ. Press, 2001

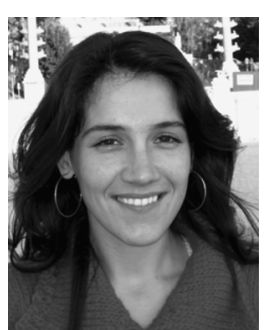

Silvana Silva Pereira (S'06) received the M.Sc. degree from the Department of Informatics, University of Oslo, Norway, in 2003. She is currently working towards the Ph.D. degree at the Signal Theory and Communications Department of the Universitat Politècnica de Catalunya-Barcelona Tech (UPC), Spain, with a grant from the Spanish Ministry of Education and Science.

In 2005, she joined the Signal Processing and Communications Group (SPCOM) at the UPC as a Research Assistant for the $\mathrm{R}+\mathrm{D}$ project $\mathrm{ECO}$ (TEC2004-04526) financed by the Spanish government. She has participated in the IST WINSOC project devoted to develop Wireless Sensor Networks with Self-Organization Capabilities for Critical and Emergency Applications. Her current research interests include distributed algorithms for wireless sensor networks and consensus.

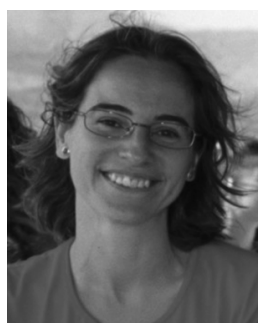

Alba Pagès-Zamora (S'91-M'03) received the M.Sc. degree and the Ph.D. degree in electrical engineering from the Universitat Politècnica de Catalunya (UPC), Barcelona, Spain, in 1992 and 1996, respectively

In 1992, she joined the Department of Signal Theory and Communications (UPC), where she became an Associate Professor in 2001. She teaches graduate and undergraduate courses related to communications and signal processing. She is author of one book chapter, eight papers in international periodic journals, and approximately 50 papers in international conferences. Her current research interests include consensus algorithms in wireless sensor networks and communication in wireless sensor networks. She has participated in six national scientific projects and one $\mathrm{R}+\mathrm{D}$ project with the European Space Agency. In addition, she has led two $\mathrm{R}+\mathrm{D}$ projects with the national industry and was the UPC's technical leader in the IST I-METRA project, the IST NEXWAY project, and the IST WINSOC project devoted to develop Wireless Sensor Networks with Self-Organization Capabilities for Critical and emergency Applications. She has also participated as an evaluator and reviewer for the European Commission (EC) of research projects in the area of Future Networks within the 6th and 7th Framework Programs. More details are provided at the author's personal Web page at http://gps-tsc4.upc.es/index.php?user=alba. 